\title{
Multidimensional Fast Multiplierless DCT Algorithm Using Ramanujan Ordered Numbers
}

\author{
Geetha K. S. and M. Uttarakumari
}

\begin{abstract}
This paper proposes a new multidimensional (m-D) multiplierless Ramanujan Numbered Discrete Cosine Transform (RDCT) based coding to improvise the coding efficiency in the visual data compression for multimedia applications. In this paper, the $m D-D C T$ with size $N_{1} \times N_{2} \times \cdots N_{m}$, where $N_{i}$ power of 2 is, can be converted into a series of one dimensional (1-D) DCT's which are evaluated using Ramanujan Ordered Numbers thereby making the algorithm multiplierless. The proposed algorithm achieves considerable savings in terms of shift operations compared with the row-column approach thus making it suitable for image and video coding applications. For a 3-dimensional input data of size $8 \times 8 \times 8$, the algorithm reduces the number of shift operations by $66 \%$ compared to multidimensional row-column approach and approximately $0.1 \%$ increase in the number of adders compared with the row-column approach. Compared with the other recently presented multidimensional DCT algorithms, the proposed one gives better result as it uses the shift operations instead of the floating-point multipliers and also achieves considerable reduction in the number of addition operation .
\end{abstract}

Index Terms-Discrete cosine transform, fast multiplierless algorithm, Ramanujan ordered number, polynomial transform

\section{INTRODUCTION}

The Discrete Cosine Transform (DCT) approaches the statistically optimal Karhunen-Loeve transform (KLT) for highly correlated data, so it is widely used in signal processing mainly for image and video compression [1], [2]. All existing digital media compression standards-JPEG, MPEG, and H.26x- are mainly based on DCT. The $m$-D DCT is chosen as an alternate approach for many image and video applications. For example, the 3-D DCT coding performs better than the motion compensated transform coding technique used in video coding standards [3]. The multidimensional transforms are also used in different areas of computer vision, HDTV video coding, and multi-frame detection [4].

Recently, many multidimensional transform algorithms have been proposed [5]-[8] which are reported to be less computationally complex compared with the conventional row-column approach [6]. In [9],[10] a recursive algorithm for generating higher order $m$-D DCT by combining the computation of $2^{m}$ identical lower order DCT architectures is presented, but it requires too many operations. The algorithm proposed in [11] converts the $m$-D DCT with size

Manuscript received June 13, 2012; revised July 12, 2012.

The authors are with Faculty, R. V. College of Engineering, Bangalore, India (e-mail: geethakomandur@gmail.com)
$N \times N \times \cdots N$ ( $\mathrm{N}$ is a power of 2) into 1-D DCT's and greatly reduces the number of operations, but it cannot support applications that require different dimensional sizes. The $m$-D algorithm in [12] is based on the factorization of the tensor product form of $m$-D DCT, uses $1 / m$ times the number of multiplications as required by the conventional row-column approach and considerable less number of addition operations compared with the row-column approach. In this method, the $m$-D data is decomposed into 1-D independent vectors based on the factorization of the tensor product, but the algorithm requires additional shifts and addition operation in both pre-processing and post-processing stages. Many polynomial transform (PT)-based algorithms for 2-D DCT [13],[14] and algorithms for multidimensional DCT [15],[16] are reported to offer reductions on the required number of operations, but these algorithms are based on the relationship between DCT and DFT and then expressing the DFT as a polynomial evaluation by polynomial transformation. The fast algorithm given by Zeng [17] can reduce the overall computational and structural complexity. The algorithm uses a technique to directly decompose the multidimensional DCT into 1-D DCT's in order to avoid operations on the complex data, but requires the floating-point multiplication operations to compute the1-D DCT's, thus being not VLSI friendly. In [18] a new PT-based algorithm is proposed to directly decompose the 2D DCT into 1-D DCT's so that operations on the complex data is not required. This algorithm uses Ramanujan ordered numbers to compute DCT's thereby replacing floating-point multipliers using shifters and adders. This method also does not require any post-addition as reported in [6] which makes the algorithm to be simpler for implementation.

In this paper, a generalized multidimensional multiplierless algorithm is proposed. The m-D data whose size is $\quad N_{1} \times N_{2} \times \cdots N_{m}$ where $N_{i}(i=1,2, \cdots m)$ is power of 2 , are directly decomposed into 1-D data. The 1-D DCT's are then computed by evaluating the cosine angles of the multiples of $2 \pi / N$. They are represented by Ramanujan ordered numbers [19] of type $2^{-l}+2^{-m}$ where $l$ and $m$ are integers. The DCT coefficients are computed using shift and addition operations only thereby avoiding the floating-point multipliers. The proposed algorithm thus achieves a considerable reduction in computational complexity as compared with the existing multi-dimensional algorithms.

This paper is organized as follows: Section 2 gives the proposed m-dimensional polynomial transforms based Ramanujan ordered number DCT. Section 3 shows the efficacy of the proposed algorithm when compared with the 
existing algorithms.

\section{MUlTidimensional DCT}

The 2-D DCT described [18] is extended for the computation of the $m$-D DCT. The $m$-D DCT can be expressed as,

$$
\begin{aligned}
& X\left(k_{1}, k_{2}, \ldots, k_{m}\right)= \sum_{n_{1}=0}^{N_{1}-1} \sum_{n_{2}=0}^{N_{2}-1} \ldots \sum_{n_{m}=0}^{N_{m}-1} x\left(n_{1}, n_{2}, \ldots, n_{m}\right) . \\
& \cos \frac{\pi\left(2 n_{1}+1\right) k_{1}}{2 N_{1}} \cdots \cos \frac{\pi\left(2 n_{m}+1\right) k_{m}}{2 N_{m}} \\
& k_{i}=0,1 \ldots, N_{i}-1 ; \quad i=1,2 \ldots, m
\end{aligned}
$$

Let us assume that $N_{i}(1,2 \ldots, m)$ are the numbers which are powers of 2 and that $N_{m}$ is the largest number in the array. Hence we can write $N_{m}=2^{t}$ and $N_{m} / N_{i}=2^{l_{i}}, \quad i=1,2 \ldots, m-1, l_{i} \geq 0$.

If the multidimensional input array could be rearranged as

$$
\begin{aligned}
& y\left(n_{1}, n_{2}, \ldots, n_{m}\right)=x\left(2 n_{1}, 2 n_{2}, \ldots, 2 n_{m}\right) \\
& y\left(N_{1}-1-n_{1}, n_{2}, \ldots, n_{m}\right)=x\left(2 n_{1}+1,2 n_{2}, \ldots, 2 n_{m}\right) \\
& \ldots \ldots . . . \\
& y\left(N_{1}-1-n_{1}, N_{2}-1-n_{2}, \ldots, N_{m}-1-n_{m}\right) \\
& \quad=x\left(2 n_{1}+1,2 n_{2}+1, \ldots, 2 n_{m}+1\right), \\
& n_{i}=0,1 \cdots, N_{i} / 2-1, \quad i=1,2 \ldots, m
\end{aligned}
$$

\section{A. Algorithm for Multidimensional DCT}

Let us define

$$
\begin{aligned}
A\left(k_{1}, k_{2}, \ldots, k_{m}\right) & =\sum_{n_{1}=0}^{N_{1}-1} \sum_{n_{2}=0}^{N_{2}-1} \cdots \sum_{n_{m}=0}^{N_{m}-1} y\left(n_{1}, n_{2}, \ldots, n_{m}\right) . \\
. & \cos \frac{\pi\left(4 n_{1}+1\right) k_{1}}{2 N_{1}} \cdots \cos \frac{\pi\left(4 n_{r-2}+1\right) k_{m-2}}{2 N_{m-2}} \\
. & \cos \left[\frac{\pi\left(4 n_{m-1}+1\right) k_{m-1}}{2 N_{m-1}}+\cdots \frac{\pi\left(4 n_{m}+1\right) k_{m}}{2 N_{m}}\right]
\end{aligned}
$$

and

$$
\begin{aligned}
& B\left(k_{1}, k_{2}, \ldots, k_{m}\right)=\sum_{n_{1}=0}^{N_{1}-1} \sum_{n_{2}=0}^{N_{2}-1} \cdots \sum_{n_{m}=0}^{N_{m}-1} y\left(n_{1}, n_{2}, \ldots, n_{m}\right) . \\
& . \cos \frac{\pi\left(4 n_{1}+1\right) k_{1}}{2 N_{1}} \cdots \cos \frac{\pi\left(4 n_{r-2}+1\right) k_{m-2}}{2 N_{m-2}} \\
& . \cos \left[\frac{\pi\left(4 n_{m-1}+1\right) k_{m-1}}{2 N_{m-1}}-\cdots \frac{\pi\left(4 n_{m}+1\right) k_{m}}{2 N_{m}}\right]
\end{aligned}
$$

Thus equation (5) can be computed as

$X\left(k_{1}, k_{2}, \ldots, k_{m}\right)=\frac{1}{2}\left[A\left(k_{1}, k_{2}, \ldots, k_{m}\right)+B\left(k_{1}, k_{2}, \ldots, k_{m}\right)\right](5)$

Let $f_{m-1}\left(n_{m}\right)$ be the least non-negative remainder of $\left[\left(4 f_{m-1}+1\right) n_{m}+f_{m-1}\right] \bmod N_{m-1}$. Similar to 2-D DCT, we can write

$$
\begin{aligned}
A\left(k_{1}, k_{2}, \ldots, k_{m}\right) & \sum_{n_{1}=0}^{N_{1}-1} \cdots \sum_{n_{m-2}=0}^{N_{m-2}-1} \sum_{f_{m-1}=0}^{N_{m-1}-1} \sum_{n_{m}=0}^{N_{m}-1} y\left(n_{1}, \cdots n_{m-2}, f_{m-1}\left(n_{m}\right), n_{m}\right) . \\
. & \cos \frac{\pi\left(4 n_{1}+1\right) k_{1}}{2 N_{1}} \cdots \cos \frac{\pi\left(4 n_{r-2}+1\right) k_{m-2}}{2 N_{m-2}} \\
. & \cos \left[\frac{\pi\left(4 n_{m-1}+1\right)\left(2^{l_{m-1}}\left(4 f_{m-1}+1\right)\right) k_{m-1}+k_{m}}{2 N_{m}}\right] \\
B\left(k_{1}, k_{2}, \ldots, k_{m}\right) & \sum_{n_{1}=0}^{N_{1}-1} \cdots \sum_{n_{m-2}=0}^{N_{m-2}-1} \sum_{f_{m-1}=0}^{N_{m-1}-1} \sum_{n_{m}=0}^{N_{m}-1} y\left(n_{1}, \cdots n_{m-2}, f_{m-1}\left(n_{m}\right), n_{m}\right) . \\
. & \cos \frac{\pi\left(4 n_{1}+1\right) k_{1}}{2 N_{1}} \cdots \cos \frac{\pi\left(4 n_{r-2}+1\right) k_{m-2}}{2 N_{m-2}} \\
. & \cos \left[\frac{\pi\left(4 n_{m-1}+1\right)\left(2^{l_{m-1}}\left(4 f_{m-1}+1\right)\right) k_{m-1}-k_{m}}{2 N_{m}}\right]
\end{aligned}
$$

and

We could define

$$
\begin{aligned}
V_{k_{1}, k_{2}, \ldots, k_{m-2}, f_{m-1}}(l) & \sum_{n_{1}=0}^{N_{1}-1} \cdots \sum_{n_{m-2}=0}^{N_{m-2}-1} \sum_{n_{m}=0}^{N_{m}-1} y\left(n_{1}, \cdots n_{m-2}, f_{m-1}\left(n_{m}\right), n_{m}\right) . \\
& . \cos \frac{\pi\left(4 n_{1}+1\right) k_{1}}{2 N_{1}} \cdots \cos \frac{\pi\left(4 n_{r-2}+1\right) k_{m-2}}{2 N_{m-2}} \\
& . \cos \left[\frac{\pi\left(4 n_{m}+1\right) l}{2 N_{m}}\right]
\end{aligned}
$$

To be the $(m-1)$ dimensional DCT with size $N_{1} \times N_{2} \cdots \times N_{m-2} \times N_{m}$. Thus equation (3) and (4) become

$$
\begin{aligned}
& A\left(k_{1}, k_{2}, \ldots, k_{m}\right) \\
& =\sum_{f_{m-1}=0}^{N_{m-1}-1} V_{k_{1}, k_{2}, \ldots, k_{m-2}, f_{m-1}}\left(2^{l_{m-1}}\left(4 f_{m-1}+1\right) k_{m-1}+k_{m}\right) \\
& B\left(k_{1}, k_{2}, \ldots, k_{m}\right) \\
& =\sum_{f_{m-1}=0}^{N_{m-1}-1} V_{k_{1}, k_{2}, \ldots, k_{m-2}, f_{m-1}}\left(2^{l_{m-1}}\left(4 f_{m-1}+1\right) k_{m-1}-k_{m}\right)
\end{aligned}
$$

Let us form the polynomial as

$$
\begin{aligned}
& B_{k_{1}, k_{2}, \ldots, k_{m-1}}(z) \\
& =\sum_{k_{m}=0}^{N_{m}-1} B\left(k_{1}, k_{2}, \ldots, k_{m}\right) z^{k_{m}}-\sum_{k_{m}=1}^{N_{m}} A\left(k_{1}, k_{2}, \ldots, 2 N_{m}-k_{m}\right) z^{k_{m}} \\
& =\sum_{k_{m}=0}^{2 N_{m}-1} B\left(k_{1}, k_{2}, \ldots, k_{m}\right) z^{k_{m}}
\end{aligned}
$$


which can be expressed as

$$
\begin{aligned}
& B_{k_{1}, k_{2}, \ldots, k_{m-1}}(z) \\
& \quad \equiv C_{k_{1}, k_{2}, \ldots, k_{m-1}}(z) z^{2^{l_{m-1} k_{m-1}}} \bmod z^{2 N_{m}}+1
\end{aligned}
$$

where

$$
\begin{aligned}
& C_{k_{1}, k_{2}, \ldots, k_{m-1}}(z) \\
& \quad \equiv \sum_{f_{m-1}=0}^{N_{m-1}-1} U_{k_{1}, k_{2}, \ldots, k_{m-2}, f_{m-1}}(z) \hat{z}^{f_{m-1} k_{m-1}} \bmod z^{2 N_{m}}+1 \\
& k_{i}=0,1,2, \ldots, N_{i}-1, \quad i=1,2, \ldots, m-1 \\
& \hat{z} \equiv z^{2^{l_{m-1+2}}} \bmod z^{2 N_{m}}+1
\end{aligned}
$$

and

$$
\begin{aligned}
& U_{k_{1}, k_{2}, \ldots, k_{m-2}, f_{m-1}}(z) \\
& =\sum_{l=0}^{2 N_{m-1}-1} V_{k_{1}, k_{2}, \ldots, k_{m-2}, f_{m-1}}(l) z^{l}
\end{aligned}
$$

which is the generating polynomial of $V_{k_{1}, k_{2}, \ldots, k_{m-2}, f_{m-1}}(l)$. The flow of the algorithm can be summarized as follows:

1) Compute the $N_{m-1}(m-1)$-dimensional DCT according to equation (5). If $(m-1)>1$, similar procedure is used to decompose the $(m-1)-\mathrm{D}$ DCT into smaller sizes. If $(m-1)=1$, the 1 -D DCT is computed using the multiplierless Ramanujan ordered number representation as in equation (16) and equation (17) of [18].

2) Compute $N_{1} N_{2} \cdots N_{m-2} \quad 1$-D polynomial transforms using equation (13), and then compute $B_{k_{1}, k_{2}, \ldots, k_{m-1}}(z)$ using equation (12).

3) $A\left(k_{1}, k_{2}, \ldots, k_{m}\right)$ and $B\left(k_{1}, k_{2}, \ldots, k_{m}\right)$ can then be computed from the definition of $B_{k_{1}, k_{2}, \ldots, k_{m-1}}(z)$ using equation (11). The Coefficients $X\left(k_{1}, k_{2}, \ldots, k_{m}\right)$ are then computed by equation (5), which requires $N-N / N_{m}-N / N_{m-1}+1 \quad$ additions where $N=N_{1} N_{2} \cdots N_{m}$.

\section{B. Computational Complexity of the Algorithm}

Computational complexity of the proposed algorithm can be explained by considering all the operations required in the three steps listed above. Hence, for the input data of size $^{N_{1} \times N_{2} \times \cdots \times N_{m}}$, the computational complexity is

$$
\begin{aligned}
& S_{m}\left(N_{1}, N_{2}, \cdots, N_{m}\right)=N_{m-1} S_{m-1}\left(N_{1}, N_{2}, \cdots N_{m-2}, N_{m}\right) \\
& A_{m}\left(N_{1}, N_{2}, \cdots, N_{m}\right) \\
& \quad=N_{m-1} A_{m-1}\left(N_{1}, N_{2}, \cdots N_{m-2}, N_{m}\right)+N \log _{2} N_{m-1} \\
& \quad+N-\frac{3 N}{2 N_{m}}-\frac{N}{N_{m-1}}+\frac{N}{N_{m-1} N_{m}}+1
\end{aligned}
$$

where $N=N_{1} N_{2} \cdots N_{m}, M_{m}\left(N_{1}, N_{2}, \cdots, N_{m}\right)$ is the number of shift operations and $A_{m}\left(N_{1}, N_{2}, \cdots, N_{m}\right)$ is the number of additions.

Considering that the computational complexity of the1-D

\begin{tabular}{|c|c|c|c|}
\hline $\begin{array}{l}\text { Oper } \\
\text { ation } \\
\mathrm{s}\end{array}$ & $\begin{array}{c}\text { 1-D RDCT } \\
{[20]}\end{array}$ & $\begin{array}{c}\text { 2-D RDCT } \\
{[18]} \\
N \times N\end{array}$ & $\begin{array}{c}\text { m-D RDCT } \\
\text { proposed } \\
N_{1} \times N_{2} \times \cdots \times N_{m}\end{array}$ \\
\hline Shifts & $N / 2 \log _{2} N$ & $N^{2} / 2 \log _{2} N$ & $\frac{1}{2} N \log _{2} N_{m}$ \\
\hline $\begin{array}{l}\text { Adde } \\
\text { rs }\end{array}$ & $\left(3 N / 2 \log _{2} N\right)-N+1$ & $\frac{5 N^{2}}{2} \log _{2} N-3 N / 2+2$ & $\begin{array}{l}\frac{3}{2} N \log _{2} N_{m}+N \log _{2} \frac{N}{N_{m}} \\
+(m-2) N \frac{3 m-5}{2} \frac{N}{N_{m}} \\
-N\left(1-\frac{1}{N_{m}}\right)\left(\frac{1}{N_{1}}+\cdots+\frac{1}{N_{m-1}}\right)+(m-1)\end{array}$ \\
\hline
\end{tabular}
DCT using Ramanujan Ordered number is $N / 2 \log _{2} N$ number of shift operations and $\left(3 N / 2 \log _{2} N\right)-N+1$ number of additions, the total number of shift operations and additions needed by the m-D DCT can be given as

$$
\begin{aligned}
& S_{m}\left(N_{1}, N_{2}, \cdots, N_{m}\right)=\frac{1}{2} N \log _{2} N_{m} \\
& A_{m}\left(N_{1}, N_{2}, \cdots, N_{m}\right) \\
& \quad=\frac{3}{2} N \log _{2} N_{m}+N \log _{2} \frac{N}{N_{m}}+(m-2) N \\
& \quad-\frac{3 m-5}{2} \frac{N}{N_{m}}-N\left(1-\frac{1}{N_{m}}\right)\left(\frac{1}{N_{1}}+\cdots+\frac{1}{N_{m-1}}\right)+(m-1)
\end{aligned}
$$

Although the proposed algorithm requires same number of additions as required by the algorithm in [17], the requirement of multipliers is completely eliminated by using the Ramanujan ordered number.

TABLE I: COMPARISON OF COMPUTATIONAL COMPLEXITY OF RDCT

TABLE II: PERFORMANCE EVALUATION OF VARIOUS MULTIDIMENSIONAL

\begin{tabular}{|c|c|c|c|c|}
\multicolumn{1}{c}{$\begin{array}{c}\text { Input } \\
\text { size }\end{array}$} & $\begin{array}{c}\text { PT } \\
\text { based } \\
\text { m-D } \\
\text { RDCT }\end{array}$ & $\begin{array}{c}\text { Recursive } \\
\text { RDCT } \\
{[18]}\end{array}$ & $\begin{array}{c}\text { Tensor } \\
\text { product } \\
{[12]}\end{array}$ & $\begin{array}{c}\text { Zeng's PT } \\
{[17]}\end{array}$ \\
\cline { 2 - 5 } & Shifts & Shifts & $\begin{array}{c}\text { Floating-poin } \\
\text { t multipliers }\end{array}$ & $\begin{array}{c}\text { Floating-poin } \\
\text { t multipliers }\end{array}$ \\
\hline $8 \times 8$ & 96 & 192 & 96 & 96 \\
$16 \times 16$ & 512 & 1024 & 512 & 512 \\
$32 \times 32$ & 2560 & 5120 & 2560 & 2560 \\
$4 \times 4 \times 4$ & 64 & 128 & 64 & 64 \\
$8 \times 8 \times 8$ & 768 & 1536 & 768 & 768 \\
\hline
\end{tabular}

TABLE III: PERFORMANCE EVALUATION OF VARIOUS MULTIDIMENSIONAL DCT ALGORITHMS IN TERMS OF ADDITION OPERATIONS

\begin{tabular}{|c|c|c|c|c|}
\hline Input size & $\begin{array}{c}\text { PT based } \\
\text { m-D } \\
\text { RDCT }\end{array}$ & $\begin{array}{c}\text { Recursive } \\
\text { RDCT } \\
{[18]}\end{array}$ & $\begin{array}{c}\text { Tensor } \\
\text { product } \\
{[12]}\end{array}$ & $\begin{array}{c}\text { Zeng's PT } \\
{[17]}\end{array}$ \\
\hline $8 \times 8$ & 466 & 6561 & 470 & 466 \\
$16 \times 16$ & 2722 & 43681 & 2554 & 2722 \\
$32 \times 32$ & 14082 & 263169 & 12786 & 14082 \\
$4 \times 4 \times 4$ & 448 & 729 & 446 & 448 \\
$8 \times 8 \times 8$ & 5600 & 24389 & 5594 & 5600 \\
\hline
\end{tabular}

\section{Simulation RESUlts}

The performance of the proposed algorithm can be evaluated by comparing the computational complexity of the algorithms with respect to the number of operations used to compute the DCT. Table I tabulates the computational complexity of the multiplierless Ramanujan ordered number DCT(RDCT) for 1-D RDCT, 2-D RDCT generalized with $\mathrm{m}-\mathrm{D}$ RDCT in terms of shift operations and addition 
operations.

Table II compares the performance of the proposed m-D RDCT with the very commonly used multi-dimensional DCT algorithms in terms of the shift/multiplication operation required. The proposed algorithm requires same number of shift operations as much as the floating-point multipliers required by the tensor product method and the Zeng's PT method. Hence the proposed algorithm is completely multiplierless. When compared with the multiplierless row-column RDCT algorithm, the proposed one reduces the complexity by $66 \%$.

Table III gives an overview of the performance of the proposed algorithm with respect to the number of addition operation required. From the table it is clear that the proposed $\mathrm{m}-\mathrm{D}$ RDCT requires no additional adders in order to compensate for the floating-point multipliers, which clearly indicates reduction in computational complexity when compared with the other multidimensional algorithms. But there is an increase in number of adders required when compared with row-column RDCT by $0.1 \%$ which is very marginal. Thus, the main advantage of the proposed algorithm is that it maintains the simplicity of the polynomial transform structure by utilizing the symmetric properties.

\section{CONCLUSIONS}

Based on the method of obtaining the multiplierless structure for computing 1-D DCT using Ramanujan ordered Number, the proposed algorithm presents a new method which generalizes to compute multidimensional DCT utilizing the benefits of polynomial transform. The use of Ramanujan ordered number completely eliminates the floating-point multiplications by shift operations only. The Polynomial transform further reduces the complexity of the algorithm to compute the multidimensional DCT. Symmetric properties of the transform are utilized to reduce the number of arithmetic operations compared with the row-column method. The requirement of the $\mathrm{M}$ being a Ramanujan ordered number is usually satisfied as the multidimensional DCT operates on a block length of either 4 or 8 or 16. Thus the proposed fast algorithm can be exploited to reduce the overall computational and structural complexity.

\section{REFERENCES}

[1] N. Ahmed, T. Natarajan, and K. R. Rao, "Discrete cosine transform,"IEEE Trans. Comput, vol. C-23, pp. 90-93, Jan. 1974.

[2] R. J. Clark, "Relation between the Karhunen-Loeve and cosine transform," in Proc. Inst. Elec. Eng. F, vol. 128, pp. 359-360, Nov. 1981.

[3] Y. L. Siu and W. C. Siu, "Variable temporal-length 3-D discrete cosine transform coding," IEEE Trans. Image Processing, vol. 6, pp. 758-763, May 1997.

[4] M. Servais and G. D. Jager, "Video compression using the three dimensional discrete cosine transform (3D-DCT)," in Proc. Symp. Commun. Signal Processing, Sep. 1997, pp. 27-32.

[5] Z. S. Wang, Z. Y. He, C. R. Zou, and J. D. Z. Chen, "A generalized fast algorithm for $\mathrm{n}-\mathrm{D}$ discrete cosine transform and its application to motion picture coding," IEEE Trans. Circuits Syst. II, Analog Digit. Signal Process, vol. 46, no. 5, pp. 617-627, May 1999.

[6] N. I. Cho and S. U. Lee, "Fast algorithm and implementation of 2-D discrete cosine transform," IEEE Trans. Circuit Syst, vol. 38, pp. 297-305, Mar. 1991.

[7] M. Bakr and A. E. Salama, "Implementation of 3D-DCT based video encoder/ decoder system," in Proc. 45th Midwest Symp. Circuits and Syst, Aug. 2002, pp. 13-16.

[8] A. Elnaggar and H. M. Alnuweiri, "A new multidimensional recursive architecture for computing the discrete cosine transform," IEEE Trans. Circuits Syst. Video Technol, vol. 10, no. 2, pp. 113-119, Feb. 2000.

[9] L. P. Chau and W. C. Siu, "Recursive algorithm for the discrete cosine transform with general length," Electron. Lett, vol. 30, pp. 197-198, Feb. 1994.

[10] Z. Wang, G. A. Jullien, and W. C. Miller, "Recursive algorithms for the forward and inverse discrete cosine transform with arbitrary length," IEEE Signal Processing Lett, vol. 1, no. 7, pp. 101-102, Jul. 1994.

[11] E. Feig and S. Winograd, "Fast algorithms for the discrete cosine transform," IEEE Trans. Signal Process, vol. 40, no. 9, pp. 2174-2193, Sep.1992.

[12] X. Chen, Q. Dai, and C. Li, "A fast algorithm for computing multidimensional DCT on small size," IEEE Trans. Signal Process, vol. 51, no. 1, pp. 213-220, Jan. 2003.

[13] P. Duhamel and C. Guillemot, "Polynomial transform computation of 2-D DCT," in Proc. ICASSP, Apr. 1990, pp. 1515-1518.

[14] J. Prado and P. Duhamel, "A polynomial-transform based computation of the 2-D DCT with minimum multiplicative complexity," in Proc. Int. Conf. Acoust, Speech, Signal Process, May 1996, pp. 1347-1350.

[15] H. J. Nussbaumer, "New polynomial transform algorithms for multidimensional DFT's and convolutions," IEEE Trans. Acoust, Speech, Signal Processing, vol. ASSP-29, pp. 74-84, Jan. 1981.

[16] H. J. Nussbaumer and P. Quandalle, "Fast polynomial transform computation of the 2-D DCT," in Proc. ICDSP, 1981, pp. 276-283.

[17] Y. Zeng, G. Bi, and A. R. Leyman, "New polynomial transform algorithm for multidimensional DCT," IEEE Trans. Signal Process, vol. 48 , no. 10 , pp. 2814-2821, Oct. 2000.

[18] K. S. Geetha and M. Uttarakumari, "New Polynomial Transform algorithm for 2-D DCT using Ramanujan Numbers," Int Conf SPCOM 2010 at IISC, July 2010.

[19] K. S. Geetha and V. K. Ananthashayana, "Fast Multiplierless Recursive transforms using Ramanujan Numbers," in Proc. IEEE International Conference on Multimedia, Signal Processing and Communication Technologies, Aligarh, India. March 2008.

[20] Y. Zeng and G. Bi, "Transforms and Fast Algorithms for Signal Analysis and Representation," Birkhuser, 2004.

[21] Y. Zeng, L. Cheng, G. Bi, and A. C. Kot, "Integer DCT's and Fast Algorithms," IEEE Signal Proc, pp. 141-14, 2000.

[22] W. H. Chen, C. H. Smith, and S. C. Fralick, "A Fast Computational Algorithm for the Discrete Cosine Transform," IEEE Trans. Comm, vol. 25, no. 9, pp. 1004-1009, Sep 1997.

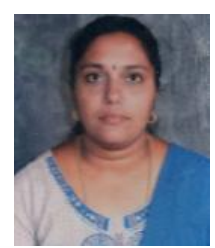

Geetha K. S. received her B.E and M.Tech in electronics engineering from National Institute of Engineering, Mysore, India, in 1991 and 1998. She is currently Assistant Professor with the Department of Electronics and Communication Engineering, R.V.College of Engineering, Bangalore, India currently working towards her $\mathrm{PhD}$ degree. Her research interests include digital signal processing, image and video processing

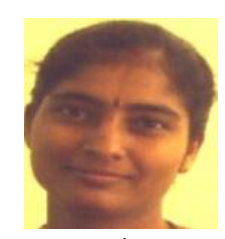

M. Uttarakumari received her B.E from Nagarjuna University in 1989 and M.Tech from Bangalore University in 1996 and Ph.D degree from Andhra university in 2007. She is currently Professor with the Department of Electronics and Communication Engineering, R.V.College of Engineering, Bangalore, India. Her current research interests include image compression, watermarking, digital signal processing. 